\title{
Varying temporal placement of response-produced stimuli in a fixed-interval schedule'
}

DAVID A. ECKERMAN and DAVID $P$. MCGOURTY, University of North Carolina at Chapel Hill, Chapel Hill, N.C. 27514

During a stimulus-available period (SAP) within a fixed-interval schedule of reinforcement (FI), each response produced a distinctive key color change (response key color changed from white to green). The influence of the temporal placement of the $S A P$ within the FI was determined for two pigeons. Responding was reduced during $S A P$ for all placements. For early placements, responding was elevated just prior to $S A P$. For late placements of the SAP responding was suppressed just prior to relatively more than during the $S A P$. Data were related to Egger and Miller's proposal that the first of a series of stimuli preceding reinforcement will be the stronger conditioned reinforcer.

A stimulus was presented following each response during a 30 -sec period within each interval of a fixed-interval 5-min schedule of food reinforcement (FI 5). During this stimulus-available period (SAP), each keypeck response of a pigeon produced a brief change in the illumination of the response key (from white light to green). Between 0 and 130 green-light presentations thus occurred during each period depending upon the response rate. In this procedure food reinforcement became available $5 \mathrm{~min}$ following the last reinforcement regardless of the intervening performance. Thus responding which produced the green light did not affect the occurrence of food reinforcement, although green lights, when they did occur, reliably preceded food reinforcement by a specified time. The temporal placement of the SAP within the 5-min interval was varied for successive phases of the experiment. To assess the effect of the stimulus at the different temporal placements, responding under the FI 5 with each placement of the SAP was compared to responding under the FI 5 when noSAP was programmed.

The present procedure derives from experiments by Farmer \& Schoenfeld (1966a, b). In the initial study, a 6-sec change in the color of a pigeon's response key occurred at various temporal positions within a FI 1-min schedule of reinforcement regardless of whether or not the pigeon responded. In the later study, the same change in key-color occurred at the change from the initial to final component of a chain FIn FIm schedule of reinforcement $(\mathrm{n}+\mathrm{m}=1 \mathrm{~min})$. In both experiments, the rates of responding under the FI schedule with light change was compared to rates during corresponding time periods when no change in key-color occurred. The effect of the stimulus was complex and was dependent upon its temporal placement within the FI.

In Farmer and Schoenfeld's second study, the pigeon was required to respond and produce the stimulus before the final, reinforced component of the chained schedule was presented. The response producing the stimulus was thus critical in producing primary reinforcement. In contrast the animal in the present study was not required to produce the stimulus. Reinforcement became available $5 \mathrm{~min}$ following the last reinforcement regardless of the intervening performance. In Farmer and Schoenfeld's first study, the stimulus was presented independent of responding and hence any relation between responding and stimulus presentations was adventitious or witnessed discriminative control. The status of the light as a conditioned reinforcer or punisher could not be assessed.

The present study assessed the influence of the response-produced green light on the FI performance when presentation of the stimulus was not necessary for the presentation of reinforcement. As such, then, the present procedure allowed assessment of the reinforcing or punishing status of the light when such responding did not alter the presentation of primary reinforcement. Also, the duration of the stimulus was reduced below Farmer and Schoenfeld's 6-sec value and the stimulus was presented for each response during an SAP so that the relative effectiveness of the first and subsequent presentations in a series could be evaluated. The present study thus allows some assessment of a proposal first made by Egger \& Miller (1962).

Egger \& Miller (1962, 1963) proposed that a stimulus is a conditioned reinforcer if it conveys information about primary reinforcement. The first rather than the subsequent members of a series of stimuli would be the most potent conditioned reinforcer according to this view, and Egger and Miller, indeed, present data supporting this view. No quarrel is presented here with their data, though some hesitancy must be admitted over the introduction of an undefined term such as "information." It is proposed, however, that the Egger and Miller result must be bounded by temporal restrictions which they leave unspecified.
In the present study, responding prior to the SAP may be used to assess the effectiveness of the first stimulus presentation of the SAP, while responding during the SAP may be used to assess the effectiveness of subsequent presentations. Further, the relative effectiveness of the first vs subsequent stimuli is studied as a function of the temporal placement of the stimuli relative to primary reinforcement.

\section{SUBJECTS}

The two Ss were White Carneaux cock pigeons, 3 years old at the end of the experiment. They were maintained at $75 \%$ of free-feeding weight and were housed in individual cages within a general vivarium. Grit and water were continuously available. Both Ss had previously been used in a discrimination study where a green light was $\mathrm{S}+$ and a red light $\mathrm{S}-($ Bird 52$)$ or the reverse (Bird 53). Several months of training on a FI 5-min schedule of reinforcement preceded the present experiment.

APPARATUS

The experimental chamber was a standard operant conditioning apparatus for pigeons with one response key located 9 in. above the floor and a feeder located directly below the key. No water was provided in the chamber. The white illumination of the key was the only light in the chamber. Each recorded peck on the key extinguished this illumination for $0.1 \mathrm{sec}$. The key light was also extinguished during operation of the feeder.

\section{PROCEDURE}

Subjects were given daily sessions of 100 reinforcements (5-sec access to mixed grain) presented according to a FI 5-min schedule of reinforcement for pecking a key illuminated with white light. After 200 sessions of training the present series of manipulations was started. The procedure was changed so that brief $(0.23-\mathrm{sec})$ key-color changes (white changed to green light) followed each response during the interval from 240 to $270 \mathrm{sec}$ in each FI 5-min interval. Fourteen-session blocks were then given in which the green light appeared following each response in the following temporal positions: $240-270$, $250-280,260-290,270-300,280-300$, and 290-300 sec. A 14-session block in which no green light was presented followed this series and served as a baseline period for measurement of the FI performance. Subsequently, the green light was again presented following responses in the 250 - to 280 -sec interval for 14 sessions. Finally, the procedure was changed so that green lights 
were presented during the $250-$ to $280-\mathrm{sec}$ intervals independent of responding. The light was presented during this interval once every $n-\sec$ where $n$ was the average rate of presentation during the last three sessions in which lights were response-contingent.

\section{RESULTS}

Figures 1 and 2 present the functions relating response rate to time within the $\mathrm{FI}$ for each bird for each experimental phase. The functions represent averages for the last three sessions of each phase. Successive functions are displaced downwards, but the

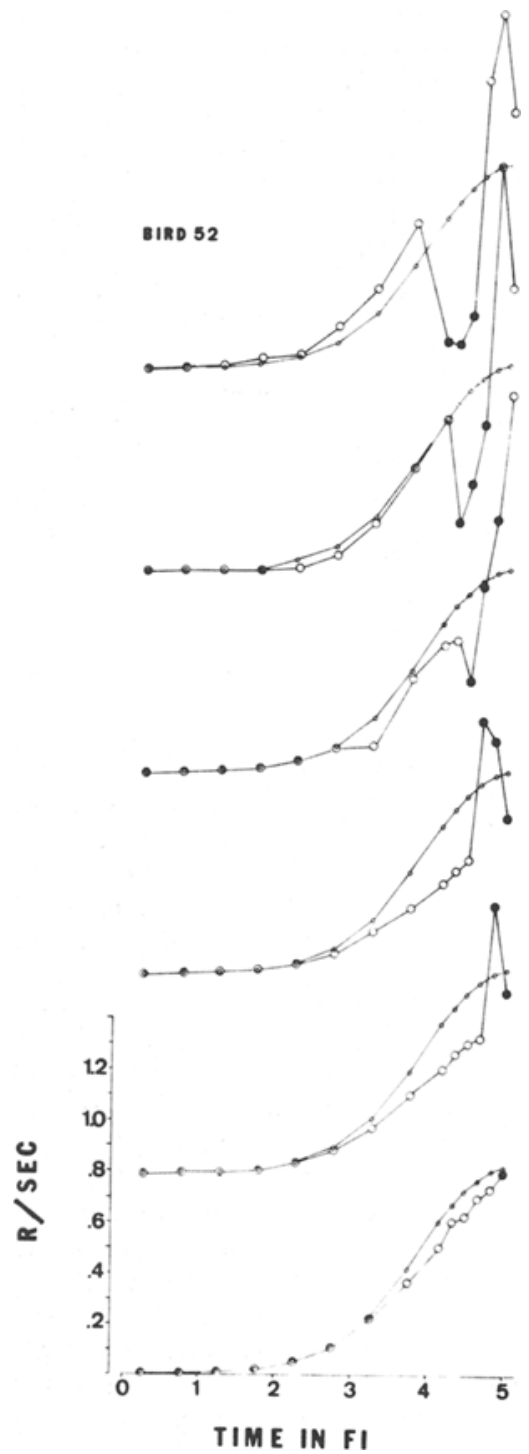

Fig. 1. Rate of responding for various positions within the FI interval for Bird 52 . Baseline FI performance is plotted (small circles), with each function characterizing an experimental phase (large circles). Functions for successive phases are displaced downward. Data points are placed at the midpoint of the interval they characterize. Filled data points represent data from SAPs (see text). baseline FI function is plotted with each to facilitate comparison.

The response-produced green lights were effective in modifying the FI performance at each temporal placement. The preponderant effect was to decrease responding both before and during SAP below that observed during the FI baseline. Certain exceptions to this effect are of interest: (1) Responding was elevated prior to SAP for the earliest SAP placement. (2) Responding was elevated following SAP and during parts of SAP for some phases for Bird 52. The fact that post-SAP rates were increased recalls the phenomenon of behavioral contrast. Inasmuch as SAPs were periods in which responding had been reduced, one might expect contrast to occur (Terrace, 1966). The presence of contrast would render the FI baseline an inappropriate reference for judging rate effects and would encourage another comparison between rate of responding at the time by (a) when this time fell within a SAP vs (b) when this time followed a SAP. The rate following SAP would include any elevation attributable to behavioral contrast and hence would be a better reference point against which reinforcing or punishing effects of the lights

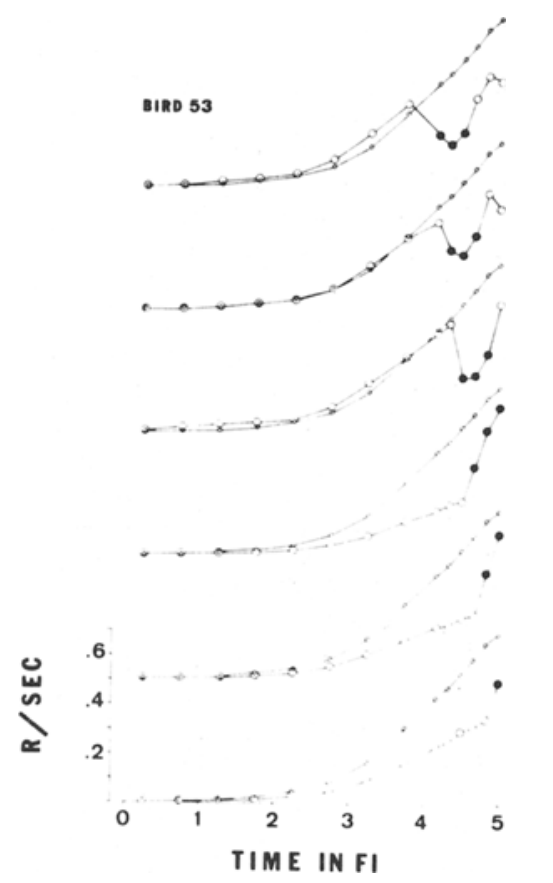

Fig. 2. Rate of responding for various positions within the FI interval for Bird 53 . Baseline FI performance is plotted (small circles), with each function characterizing an experimental phase (large circles). Functions for successive phases are displaced downward. Data points are placed at the midpoint of the interval they characterize. Filled data points represent data from SAPs (see text). might be assessed. These intraprocedural comparisons show rate to be consistently lower during SAP for Bird 52. The lights during SAP may thus be considered as punishers for both birds.

The major effect of the lights was thus to suppress responding which produced them. Responding prior to the SAP was, however, elevated for the early placement of SAP. Such a result might be summarized by saying that the initial green light was a conditioned reinforcer while subsequent lights were conditioned punishers (using Azrin and Holz's 1966 definition of punishment). The argument may be extended as follows: For all placements of SAP in which the SAP did not terminate in reinforcement, responding prior to SAP was either higher than baseline or relatively less suppressed then responding during SAP. We may summarize this result by saying that the initial green light was more reinforcing than subsequent ones. But the argument is reversed for SAPs which terminated in reinforcement. Responding prior to SAP was suppressed relatively more than responding during SAP and thus initial green lights were relatively less reinforcing than subsequent ones. The relative influence of initial and subsequent green lights was thus reversed for different temporal placements of SAP.

Intra-SAP changes were consistent for Bird 53. Responding increased in rate within SAPs indicating that later green lights were less effective punishers than early ones at all temporal placements of SAP. This effect also characterizes early SAP placements for Bird 52. Rate changes at late placements of SAP were complicated, however, by Bird 52's tendency to slow responding toward the end of the FI. This slowing was less pronounced when SAP was placed at the end of the FI than when SAP was placed earlier. This reduction in the slowing may represent an effect comparable to that found for Bird 53. However, since the slowing is even less dramatic in the baseline FI than in late-placement SAPs, the characterization of intra-SAP changes for Bird 52 is perhaps best left moot.

During the last phase of the experiment, response-produced lights were compared in effect to response independent lights. Data from this phase are presented in Fig. 3 as functions relating response rate to time within the FI. The functions represent averages for the last three sessions of each part of this phase.

The effect of removing the response contingency differed for the two birds. For Bird 52, response-independent lights did not suppress responding indicating that the light's effect was more that of a punisher than that of a discriminative stimulus. For Bird 53, response-independent lights reduced responding as much as (if not more 


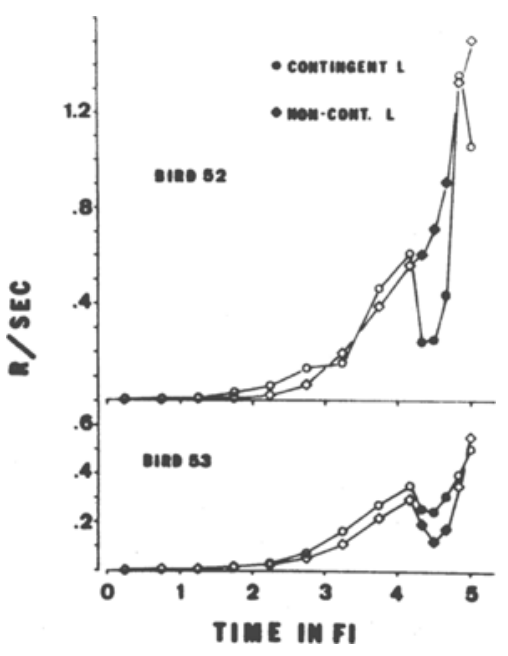

Fig. 3. Rate of responding for various positions within the FI interval for Birds 52 and 53. The circles represent data from a procedure in which response-produced lights were presented in SAPs (filled circles) and diamonds represent data from a procedure in which response-independent lights were presented in SAPs (filled diamonds). For definition of SAP, see text.

than) response-produced lights. It is thus possible that the lights exercised a discriminative rather than punishing influence. Unfortunately, however, this conclusion is not forced since an adventitious relation between responding and the lights could have acted to reduce responding. That is, the suppressed responding could have been superstitious.

\section{DISCUSSION}

The major effect of the responseproduced stimuli was to reduce responding which produced them. The stimuli were thus conditioned punishers by Azrin \& Holz's definition (1966). The second effect of the stimuli was, however, to elevate responding just prior to the SAP either in absolu te terms (SAP from $240.270 \mathrm{sec}$ ) or relative to responding during the SAP (SAPs from $250-280$ and $260-290 \mathrm{sec}$ ). Such an effect suggests that the initial response-produced stimulus acts either as a conditioned reinforcer (240- to 270-sec SAP) or at least as a less effective conditioned punisher (250to 280- and 260- to 290-sec SAPs) for certain SAP placements. Such a result is similar to that of Egger \& Miller (1962, 1963).

Data which are contrary to the predictions of Egger and Miller are as follows: (1) the reduction in rate of responding prior to SAPs for placements in which the SAP terminated in reinforcement indicates that initial response-produced stimuli were less effective than subsequent stimuli in maintaining behavior for these SAP placements; (2) the tendency to scallop within SAP intervals indicates that later stimuli were more effective than initial in maintaining behavior. These disagreements suggest that closer attention to temporal and procedural details than implied by the word "information" will be required to adequately characterize the relative effectiveness of members of a series of stimuli as conditioned reinforcers.

In the present study the responseproduced stimuli were paired with food at delays varying from 0 to $60 \mathrm{sec}$. Although the relative effect of initial and subsequent stimuli in a series changed across this range as described above, the predominant effect throughout the range was response suppression. The lights were punishers. Such an effect might have been expected from the role of the lights as $\mathbf{S}$-'s acting as "clock stimuli" in the FI interval. Indeed, the influence of the SAPs is almost identical with the influence of Farmer \& Schoenfeld's (1966a) clock stimulus placed at various positions within the FI. Such an expectation derives from the view that the green lights were exerting discriminative control over responding. This interpretation is, however, rendered suspect for Bird 52 by the lack of discriminative control exerted by responseindependent stimuli in the last phase of the study, though the interpretation is moot for Bird 53.

The SAP of the present experiment had an effect very like that of a clock stimulus in an FI schedule of reinforcement (Farmer \&
Schoenfeld, 1966a) and like that of a stimulus marking a change from initial to final component of a chain $\mathrm{FI}_{n 1} \mathrm{FI}_{\mathrm{m}}$ schedule of reinforcement (Farmer \& Schoenfeld, $1966 \mathrm{~b}$ ). Such a similarity is at once hopeful and discouraging. It is hopeful since it suggests great generality of the influence of delayed reinforcement on the influence of a stimulus. It is discouraging since it suggests that the response contingency imposed in this study and that of Farmer \& Schoenfeld (1966b) may not be as critical as we would hope. The fact that removal of the contingency did affect the performance of one bird of the present study holds some hope, however, that the response contingency is important. If the chained schedule procedure is going to be useful in the analysis of conditioned reinforcement effects vis a vis discriminative stimulus effects, a special role for the response contingency is required.

\section{REEERENCES}

AZRIN, N. H., \& HOLZ, W. C. Punishment. In W. K. Honig (Ed.), Operant behavior: Areas of research and application. New York: AppletonCentury-Crofts, 1966.

LGGER, M. D. \& MILLER, N. F. Secondary reinforcement in rats as a function of information value and reliability of the stimulus. Journal of Experimental Psy chology. $1962,64,97-104$

EGGFR, M.D., \& MIL LER, N. E. When is a rewart reinforcing? An experinental study of the information hypothesis. Journal of Comparative \& Physiological Psychology, 1963, 56. 132-137.

FARMER, J., \& SCHOENFELD, W. N. Varying temporal placement of an added stimulus in a fixed-interval schedule. Journal of the Experimental Analysis of Behavior, 1966a, 9, 142-148.

FARMER, J., \& SCHOENF ELD, W. N. The effect of a response-contingent stimulus introduced into a fixed-interval schedule at varying temporal placement. Psychonomic Science, $1966 \mathrm{~b}, 6,15-16$.

TERRACE, H. S. Stimulus control. In $w, k$. Honig (Ed.), Operant behavior: Areas of research and application. New York: Appleton-Century-Crofts, 1966. NOTE

1. This work was supported by Grant 324-1 AC 1454) from the Research Countil of the University of North Carolina and by Grant MII07534 from the Institute of Mental Health, Public Health Service to Dr. Marcus B. Waller.

\section{Notes \& News}

The American Journal of Psychology has decreased the time required for editorial decisions and now has a publication lag of only a few months. Manuscripts may be sent to Lloyd G. Humphreys, Department of Psychology, University of Illinois, or to one of the other principal editors listed in the journal.
The Tenth Annual Meeting of the Psychonomic Society will be held at the Chase-Park Plaza Hotel in St. Louis, Nov. 6, 7, 8, 1969. A Call for Papers has been mailed to members. Those who are not members may present papers when sponsored by a member. Hence, a non-member who wishes to present a paper should contact a member, secure a form, fill it out and have it signed by the member-sponsor.
Deadline for Abstracts will be August 1, 1969. Abstracts as well as requests for further information on the meetings should be sent to Psychonomic Society, Inc., 1200 West 34th St., Austin, Texas, 78705. The complete program of the meetings will be published in September as a supplementary issue to Psychonomic Science. It will, of course, be distributed to members who are not subscribers to this Journal. 